\title{
High Temperature Wireless Sensor Network Monitoring System for Coalmine Fire
}

\author{
Margaret Richardson Ansah, Yan Shu, Cao Qing Hua \\ School of Computer Science and Telecommunication Engineering, Jiangsu University, Zhenjiang, 212013
}

\begin{abstract}
Wireless Sensor Network node for monitoring coal seam combustion has been designed and implemented for surface coalmine. A wide range temperature WSN node has been implemented by integrating JN5139 microcontroller; MAX31855 temperature sensor and K-type thermocouple as the main components of the node. Data is remotely sensed from source nodes and transmitted to the sink node using the IEEE 802.15.4 standard with Zigbee protocol for the communication between nodes. Sensing temperature, data processing and transmission of data are software-controlled by the JN5139 microcontroller, which is interfaced with the MAX31855 through SPI. Data is transmitted over the internet through GPRS for remote access. The node is stable and robust enough to withstand harsh environmental condition on the coalfield. The node is able to provide readings of temperature range from $-40^{\circ} \mathrm{C}$ to $1300^{\circ} \mathrm{C}$.
\end{abstract}

Keywords: High temperature; monitoring; wireless sensor network; Coalmine; Remote access

\section{INTRODUCTION}

Over the past few years, there has been a considerable interest in the research and development of wireless sensor network (WSN) nodes for industrial use and environmental monitoring. The use of sensors to corporately monitor physical or environmental quantities such as temperature, pressure, motion, pollutants, irrigation, habitat, wildfires, volcano, vibration etc. has been widely studied [1-9] to make monitoring relatively easier. These developments have also reduced labor cost and improved efficiency in data monitoring.

However, there are still many applicable areas that have not been fully explored. Due to the fact that WSN deployment is application specific, specific designs are required for specific applications such as coal mines. Related works done on application of WSN in coalmines differ substantially in goals and scope from this work. Li and Liu[10], Li-Min et al[11], Bian[12] and Wang[13] focused on underground safety in the coalmines. In [14], Yang and Huang worked on the security aspect of coalmine. Other works that monitored temperature however did not work on coalmines. For instance, Lloret et al[15] and Yu et al[16] detected forest fire which did not require measurement of high temperature. In [17] and [18], Antion-Santoni et al and Manolakos et al simulated forest fire which are theoretical. Talzi et al [19] and Li et al[20] monitored only low temperature. Off-the-shelf sensor nodes on the market such as in [21-23] do not meet the application requirements of coalfields fire in monitoring very high and very low temperatures. $\mathrm{Li}[24]$ and $\mathrm{Cao}[25]$ used MAX6675 sensor, which is limited to measuring temperatures from $0^{\circ} \mathrm{C} \sim 1023^{\circ} \mathrm{C}$. The scope of this article is to measure very high temperature (up to $1600^{\circ} \mathrm{C}$ ) of coalfield seam combustion and very low (-40) when the fire is put off during winter season and transmitting the data over a long distance of up to 300 meters.

This article describes the design and implementation of a WSN monitoring system based on Zigbee for measuring very high and low temperature of coalfield fire and any other industrial use that require to measure very low and very high temperature remotely. It combines sensing, control automation, information processing, digital and wireless transmission, and information storage technologies to make remote monitoring highly efficient and effective.

\section{DESIGN AND IMPLEMENTATION}

The use of WSN with Zigbee technology dispenses with the associated high cost of wiring. The entire system architecture consists of a group of sensor nodes, a base station that is PC, in this case. Real-time data and time-series are achieved through client/server mode data management software. JN5139 micro-processor is adopted as the core component for the wireless sensor node and the Zigbee protocol is used for the communication between nodes. 
High Temperature Wireless Sensor Network Monitoring System

\section{A. System Architecture}

The monitoring system architecture consists of two main parts, a wireless monitoring system and a remote data management base station. The wireless monitoring system is made up of a sink node and several router nodes which forward sensed data to the sink node. The router nodes are arranged in a star topology around the sink node depending on the area under monitoring. The design requirements of the wireless sensor monitoring network nodes are as follows: continuous sensing of real-time temperature data between the range of $-40^{\circ} \mathrm{C}$ and $1300^{\circ} \mathrm{C}$, periodic transmission of monitored data, roll and display monitoring data on a PC at a base station, remote accessibility of monitored data, robustness and scalability of nodes and network.

\section{1) Hardware Structure of WSN Node}

A typical WSN consists of autonomous wireless sensing, processing and transmitting nodes that are self-organized to form a network. The hardware aspect of the node has four main functional units. It is made up of the power module, sensing module, processing module and the communication module as shown in figure 1.

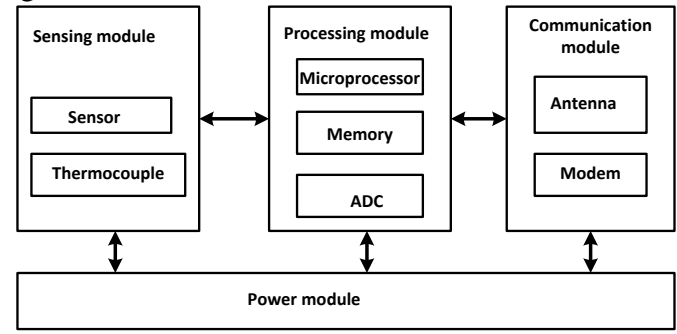

Figure 1: WSN node modules

a).Sensing Module: The achievement of the objective of this paper solely depends on the ability and accuracy of the sensing module. To be able to monitor very high and low temperature, the sensing module becomes the most important part of the whole WSN node. The selection of the sensor contributes much to the achievement of the goal. Parameters considered in the selection of the temperature sensor were the temperature sensing range, the temperature resolution, operating temperature range and the processor speed. These parameters led to the choice of MAX31855 as the sensor that met the parameter requirements of this paper. There are different versions with varying sensing ranges of MAX31855. However, MAX31855K was chosen for this work due to familiarity with Ktype thermocouple. K-type thermocouple was used to sense the temperature.

b).The Processing and Communication Module: The Jennic microcontroller board was used for the implementation. JN5139 node has some desirable feature such as low power consumption, high stability and robustness that met the hardware requirement of this work. JN5139 has a 32-bit RISC processor, 192kB of ROM and $96 \mathrm{~Kb}$ of RAM which support the storage of the system software, including protocol stacks and application data. It also integrates $2.4 \mathrm{GHz}$ IEEE802.15.4 compliant transceiver and Zigbee wireless which is responsible for the wireless communication between nodes. An antenna is connected to boost signal strength. The power module consists of a $6 \mathrm{~V}$ rechargeable battery and a solar cell for continuous power supply. It integrates voltage stabilizing unit.

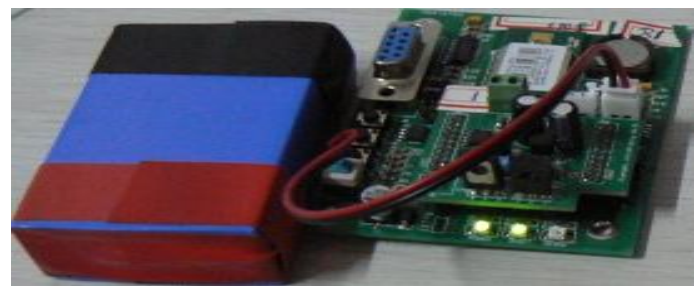

Fig 2: Complete node

\section{SYSTEM SOFTWARE DESIGN AND DATA PROCESSING}

The JN5139 uses Zigbee protocol stack to support its application development. The software digitally controls processes such as setting SPI communication interface between JN5139 microcontroller and MAX31855, reading data from the thermocouple and transmitting to the serial interface of the MAX31855, shifting of data to the microcontroller after A/D conversion and the wireless transmission of data. The JN5139 sensor node uses three main network topology types namely star, mesh and cluster tree. For experimental purpose, the network formation of the node is a simple star topology with a single hop router to coordinator data transmission as shown in figure 5. 
The network consists of a PAN coordinator, six router nodes which act as end devices. The main function of the application which is coded in C programming language on Jennic Code blocks software is shown in state diagram in figure 4.

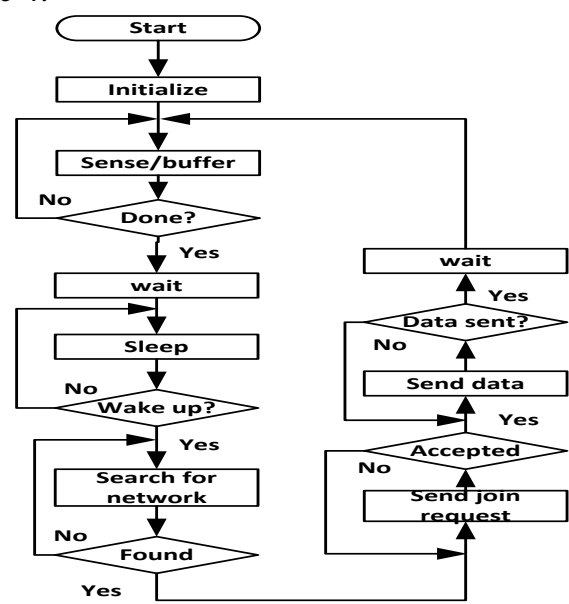

Figure 3: a) Router node process

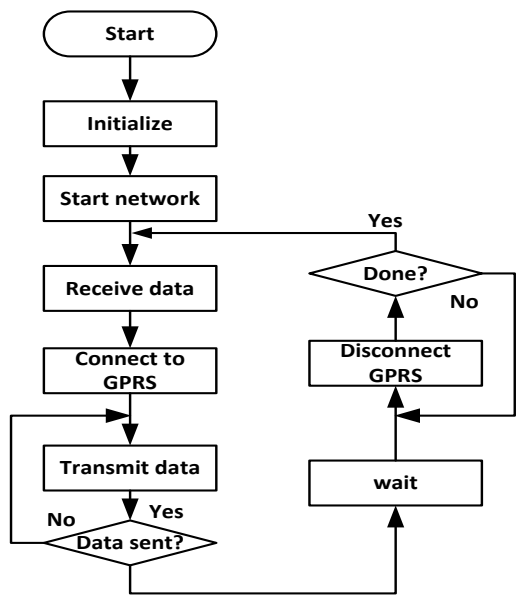

b) Coordinator node process

The node operates as follows: Each node on the network has an identification number and the coordinators are numbered according to fire areas, therefore it is easy to know exactly what goes on at each fire area in real time. The MAX31855 through the thermocouple senses 32-bits temperature in a form of voltage signals and converts the signal into decimal values. After conversion, the data is transmitted through the wireless RF module to the coordinator node as shown in figure 3a. The coordinator forwards the data to the base station and the web through the GPRS. EP220P GPRS[26] was used to transfer data over the internet. An event is generated from the instruction in a program which automated the data transfer over the internet. The coordinator node can also act as a router node by sensing temperature of fire area. The software of the remote data management platform is designed in C\# language and based on SQL Server for the display-part of the wireless monitoring system. The data collected from different fire areas is shown in Figure 9. The procedure does not only transmit temperature data, it also includes node power voltage, node address, and destination node address which are necessary for data classification.

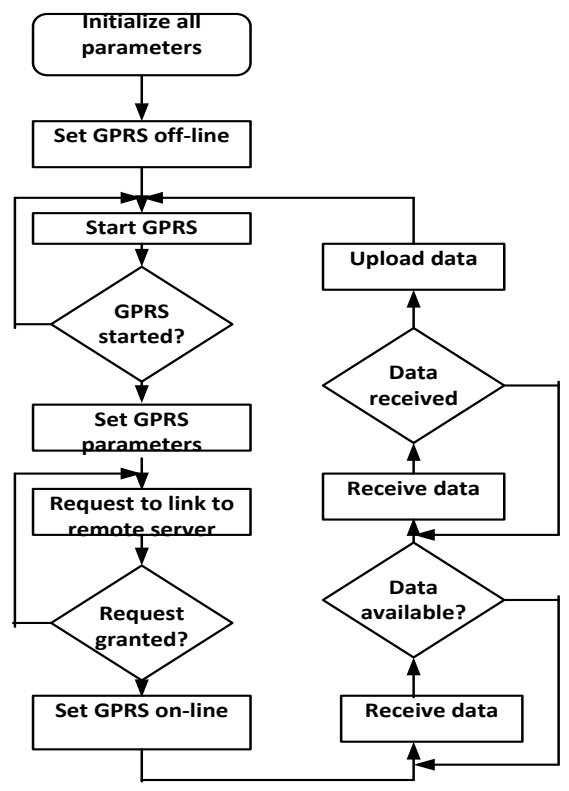

Figure 5: Working cycle of a GPRS 
Each node, as shown in figure 5, is enclosed in a metallic container to shield it from harsh environmental conditions. The enclosure is perforated to keep the node ventilated whiles preventing rain water from entering. Each node is mounted on a pole with the solar panel as shown in figure 6 to keep it from mechanical damage.

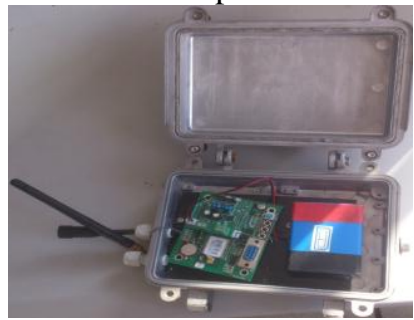

Figure 5: Cased node

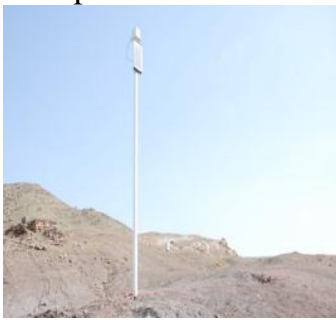

Figure 6: Field set up

The need for the node to operate unattended on the coalfield was factored into the design to make it robust. The environmental conditions on the coalfield are very harsh during winter and summer times coupled with thunder storms during autumn. The nodes are therefore designed and programmed to be robust in terms of self organization and its resistance to these environmental conditions to enable it to operate unattended for a long period of time. The addition of solar panel to the design charges the battery thereby keeping the node constantly powered. The sink node is placed in a cabin as shown in Figures 7 a) and b) to make it visible trucks drivers working on the field.

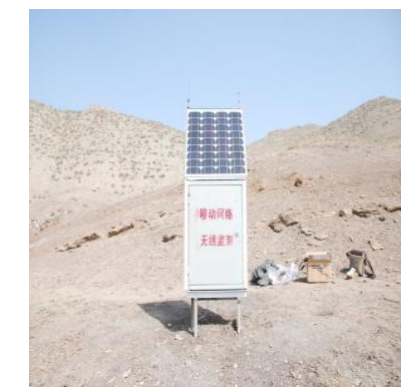

a)

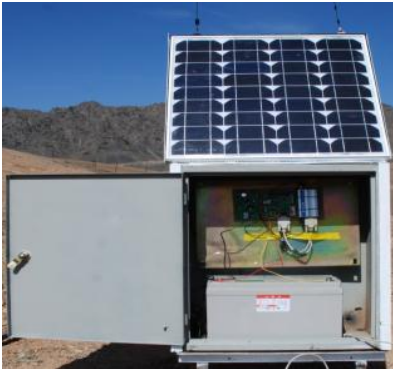

b) Sink node in a cabin

Figure 7: Monitoring system field overview

\section{A. Monitoring System Structure and Remote Data Access}

The coalfield is divided into sections called fire areas. Each fire area is assigned six nodes, five of which act as end devices called router nodes and one as a coordinator node which serve as a gateway to the group. The nodes are spread around fire area with a distance of $50 \mathrm{~m}$ between nodes. Each coordinator node is connected to a GPRS which in turn sends data to the base station and the internet. The data can then be accessed remotely. The network is a single hop router to coordinator which is connected to the base station. With the exception of the coordinator, nodes are able to join or leave the network without affecting the flow of data.

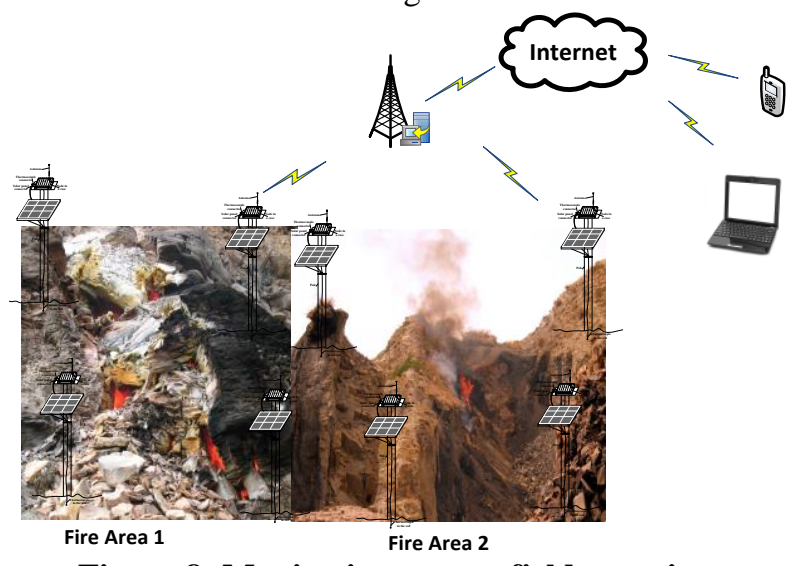

Figure 8: Monitoring system field overview 


\section{RESULT AND DISCUSSION}

The performance of a system depends on its accuracy therefore the high temperature wireless monitoring network has undergone series of tests in the laboratory. The tests include placing the thermocouple probe into boiling water, hot flame and at room temperature. The wireless monitoring network has continuously operated in the Lab and no abnormal phenomenon has occurred. In order to test the nodes the real-time sensing and remote accessibility, the nodes have been tested on the coalfield, with distance between nodes as $50 \mathrm{~m}$. The nodes were set to acquire and send data every 2 minutes and sleep for 5 minutes, the whole system operated more than 15 days. Data packet delivery rate was $100 \%$ with no packet error. However, when it rained heavily with thunder storms, most of the node broke down which will be investigated in future work. When a star network topology was used, data from a particular fire area is completely loss when a coordinator or a GPRS broke down. However, mesh network topology can be used to allow multi-hop communication between nodes and coordinator and provide alternative paths to the sink node. Sensor readings was compared with readings from a digital thermometer and accuracy was found to be $\pm 2 \%$. Figure 9 shows the temperature, ambient temperature and humidity with time series.

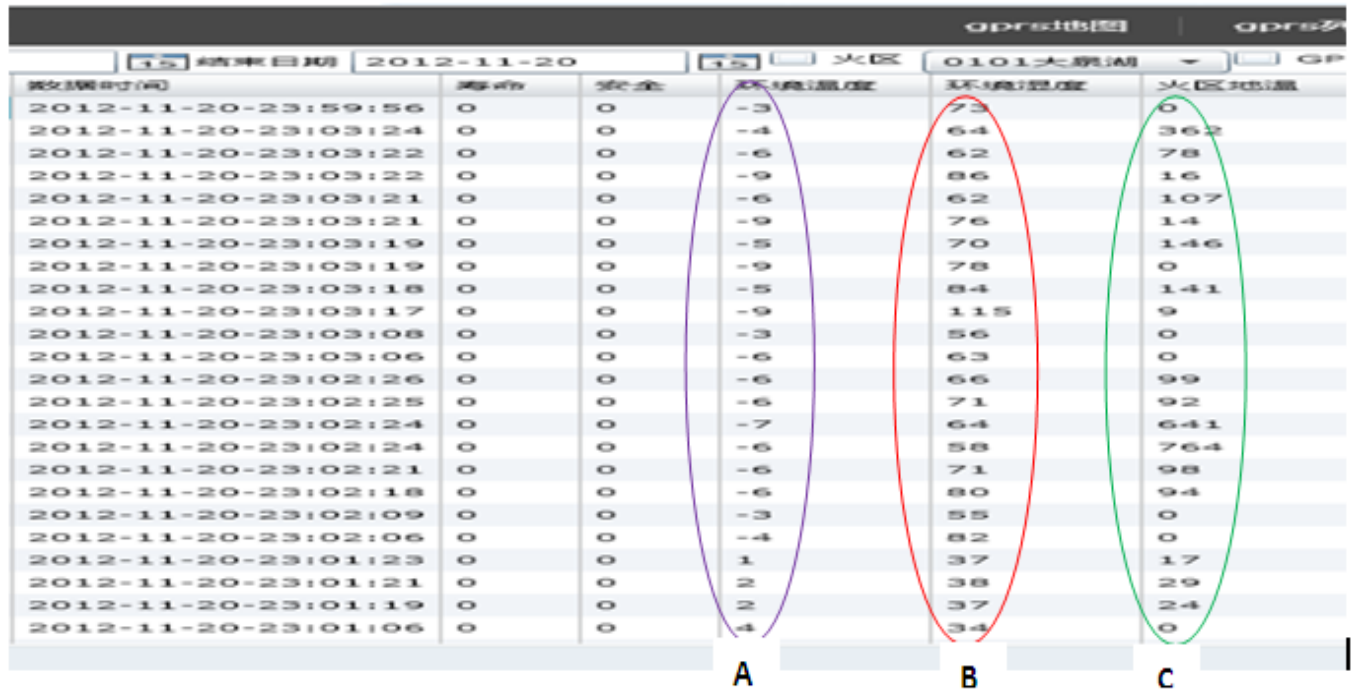

A- Ambient temperature, B- humidity, $\mathbf{C}$ - coal fire temperature GPRS data from all the fire areas Figure 9: Field results

\section{CONCLUSION}

Our contribution in this article is to use WSN application in the core process of coal mine fire combustion very high temperature monitoring in terms of sensing, processing and transmission and making the data remotely accessible. The design of combining JN5139 microcontroller interfaced with MAX31855 and K-type thermocouple make wireless sensor network nodes that can be used to monitor very high and low temperature possible. It expands the range of temperature than can be sensed and transmitted by existing wireless sensor network nodes. Applications that need to monitor very high temperature such as coalfields and other application that needs to monitor very low temperature such as frozen or very cold habitat can also use it. It has the advantage of accessing data away from the field. Unique identification of nodes helps in easy identification of faulty nodes and its maintenance. The nodes are designed to withstand harsh environmental conditions and are able to work unattended for a long period of time. It will greatly improve performance and efficiency by way of providing real-time data to field managers in order to know when to go to the coalfield. The node is capable of monitoring temperature range of $-40 \sim 1300$ in short term and a long term range of $-40 \sim 1000$ due to the limitation of K-type thermocouple. However, other versions of MAX31855 such as R, S and T with their respective thermocouples can be used to monitor much higher temperature than MAX31855K.

\section{Acknowledgement}

This research is supported by the Science and Technology Assistance in Xinjiang Projects of Xinjiang Uygur Autonomous Region, China (201191210

\section{DECLARATION}


The author(s) declare(s) that there is no conflict of interests regarding the publication of this article.

\section{References}

[1]. M. I. Neace. A. M. Neacă, "High Temperatures Measurement in Industrial " Internet Computing, IEEE, vol. 978-1-4673-1810, 5/12/ 20122012.

[2]. K. Arshak and E. Jafer, "A wireless sensor network system for pressure and temperature signals monitoring," in Industrial Electronics, 2007. ISIE 2007. IEEE International Symposium on, 2007, pp. 1496-1501.

[3]. R. Jedermann, R. Schouten, A. Sklorz, W. Lang, and O. Van Kooten, "Linking keeping quality models and sensor systems to an autonomous transport supervision system," in Cold Chain Management-2nd international Workshop, Bonn, 2006.

[4]. R. P. a. A. M. Kavi K. Khedo, "A Wireless Sensor Network Air Pollution Monitoring System," International Journal of Wireless and Mobile Networks (IJWMN), vol. Vol.2, May 2010.

[5]. X. Xiang, "Design of fuzzy drip irrigation control system based on ZigBee wireless sensor network," in Computer and Computing Technologies in Agriculture IV, ed: Springer, 2011, pp. 495-501.

[6]. R. Szewczyk, J. Polastre, A. Mainwaring, and D. Culler, "Lessons from a sensor network expedition," in Wireless Sensor Networks, ed: Springer, 2004, pp. 307-322.

[7]. B. Son, Y.-s. Her, and J. Kim, "A design and implementation of forest-fires surveillance system based on wireless sensor networks for South Korea mountains," International Journal of Computer Science and Network Security (IJCSNS), vol. 6, pp. 124-130, 2006.

[8]. G. Werner-Allen, K. Lorincz, M. Ruiz, O. Marcillo, J. Johnson, J. Lees, and M. Welsh, "Deploying a wireless sensor network on an active volcano," Internet Computing, IEEE, vol. 10, pp. 18-25, 2006.

[9]. M. Bocca, E. Cosar, J. Salminen, and L. Eriksson, "A reconfigurable wireless sensor network for structural health monitoring," Proceedings of International Society for Structural Health Monitoring of Intelligent Infrastructures (ISHMII-4), Zurich, July 22-24, 2009.

[10]. M. Li and Y. Liu, "Underground coal mine monitoring with wireless sensor networks," ACM Transactions on Sensor Networks (TOSN), vol. 5, p. 10, 2009.

[11]. Y. Li-min, L. Anqi, S. Zheng, and L. Hui, "Design of monitoring system for coal mine safety based on wireless sensor network," in Mechtronic and Embedded Systems and Applications, 2008. MESA 2008. IEEE/ASME International Conference on, 2008, pp. 409-414.

[12]. J. Bian, "Application of the wireless sensor network based on ZigBee technology in monitoring system for coal mine safety," in Computer, Mechatronics, Control and Electronic Engineering (CMCE), 2010 International Conference on, 2010, pp. 204-206.

[13]. Y.-f. WANG, Z.-b. ZHANG, and C.-j. LI, "Wireless sensor network applied to coal mine gas monitoring and measuring system [J]," Coal Science and Technology, vol. 6, pp. 34-36, 2007.

[14]. W. Yang and Y. Huang, "Wireless sensor network based coal mine wireless and integrated security monitoring information system," in Networking, 2007. ICN'07. Sixth International Conference on, 2007, pp. 13-13.

[15]. J. Lloret, M. Garcia, D. Bri, and S. Sendra, "A wireless sensor network deployment for rural and forest fire detection and verification," Sensors, vol. 9, pp. 8722-8747, 2009.

[16]. L. Yu, N. Wang, and X. Meng, "Real-time forest fire detection with wireless sensor networks," in Wireless Communications, Networking and Mobile Computing, 2005. Proceedings. 2005 International Conference on, 2005, pp. 1214-1217.

[17]. T. Antoine-Santoni, J.-F. Santucci, E. De Gentili, X. Silvani, and F. Morandini, "Performance of a protected wireless sensor network in a fire. Analysis of fire spread and data transmission," Sensors, vol. 9, pp. 5878-5893, 2009.

[18]. E. S. Manolakos, D. Manatakis, and G. Xanthopoulos, "Temperature field modeling and simulation of wireless sensor network behavior during a spreading wildfire," in Proceedings of the 2008 European Signal Processing Conference (EUSIPCO 2008), Lausanne, Switzerland (August 2008), 2008.

[19]. I. Talzi, A. Hasler, S. Gruber, and C. Tschudin, "PermaSense: investigating permafrost with a WSN in the Swiss Alps," in Proceedings of the 4th workshop on Embedded networked sensors, 2007, pp. 8-12.

[20]. C.-M. Li, C.-C. Nien, J.-L. Liao, and Y.-C. Tseng, "Development of wireless sensor module and network for temperature monitoring in cold chain logistics," in Wireless Information Technology and Systems (ICWITS), 2012 IEEE International Conference on, 2012, pp. 1-4.

[21]. $\quad$ N. L. U. Ltd, "Jennic Wireless Microcontrollers."

[22]. T. I. TI, "CC2530 user guide and specifications," 05-19-2010 2010.

[23]. N. I. NI, "NI WSN-3202 user guide and specifications for NI Wireless Sensor Network 4-Channel, 16-Bit Analog Input Node " p. 25.

[24]. S.-R. Li, S. Yan, Q.-H. Cao, and Z.-J. Chen, "Design and Development of Wireless Sensor Network High Temperature Monitoring Node," Jisuanji Gongcheng/ Computer Engineering, vol. 38, 2012.

[25]. Q.-H. C. Cao, Zu-Jue; Li, Shan-Rong; Yan, Shu, "Wireless sensor network monitoring node and method for 130o 1000oC high temperature of fire area in coalfield," 12-10-2011 2011.

[26]. EP220P GPRS DTU User’s Guide.2002.Shanghai Eplus Smart Equipment Co.,Ltd,3. 